\title{
Influence of atomic mixing and preferential sputtering on depth profiles and interfaces
}

\author{
Z. L. Liau, B. Y. Tsaur, and J. W. Mayer \\ California Institute of Technology, Pasadena, California 91125 \\ (Received 28 October 1978; accepted 18 December 1978)
}

\begin{abstract}
Atomic mixing and preferential sputtering impose a depth resolution limit on the use of sputter sectioning to measure the composition of metal-semiconductor interfaces. Experimental evidence obtained with the $\mathrm{Pt}-\mathrm{Si}$ system is used to demonstrate ion-induced atomic mixing and then its effect on sputter etching and depth profiling. Starting with discrete layer structures, a relatively low ion dose $\left(\gtrsim 3 \times 10^{15} \mathrm{~cm}^{-2}\right)$ first produced a mixed surface layer with a thickness comparable to the ion range. Higher ion doses then result in successive sputter etching and continual atomic mixing over a constant surface layer thickness. A model is developed that is based on a sputter-removal (including preferential sputtering) of atoms at the surface and a uniform mixing of atoms over a constant thickness. The model predicts the influences of atomic mixing and preferential sputtering on the depth profiling of thin-film structures and interfaces.
\end{abstract}

PACS numbers: $81.15 . J j, 81.15 . \mathrm{Cd}, 92.0 \mathrm{~N} . \mathrm{c}, 81.90 .+\mathrm{c}$

\section{INTRODUCTION}

The most commonly used techniques for studying material aspects of thin films interfaces are Auger electron spectroscopy (AES), secondary ion mass spectroscopy (SIMS) and Rutherford backscattering spectrometry (RBS). The RBS technique can analyze interfaces in a nondestructive and quantitative manner. However, its depth resolution is limited by the intrinsic energy resolution of the detectors. With the commonly used solid-state detectors, the corresponding depth resolution is $\sim 200 \AA$ for $2-\mathrm{MeV}^{4} \mathrm{He}^{+}$backscattering. Improvements can be made by using a glancing angle incidence of the analyzing ion beam. For example, with $80^{\circ}$ away from normal incidence, a depth resolution of $\sim 40 \AA$ can be achieved at the surface. However, this improved depth resolution is lost below the surface due to straggling in the energy loss process of the ions in the solid. ${ }^{1}$ The degradation in resolution becomes worse for deeper regions in the material, regions where the interfaces are generally located.

Both AES and SIMS are highly surface-sensitive, because the escape depths of Auger electrons and secondary (or sputtered) ions are generally $\leqslant 10 \AA .^{2}$ Sputter-etching with ions of $1 \sim 10 \mathrm{keV}$ is generally used for surface cleaning and in-depth analysis. Problems associated with the sputtering phenomena can, therefore, affect the actual depth resolution. In addition to the sputter-induced surface roughness and composition changes, numerous experiments have shown that the ions used for sputtering can "mix" atoms over depths comparable to the ion range. ${ }^{3-11}$ Since these depths are usually greater than the escape depths of Auger electrons and secondary ions, AES and SIMS techniques are essentially analyzing the "mixed" layers.

In the present paper, we first present some recent work on ion-induced atomic mixing, emphasizing the "effectiveness" of the mixing process and its consequences. We then develop a model to understand the influences of atomic mixing and preferential sputtering on the thin-film and interface analyses.

\section{ION-INDUCED ATOMIC MIXING}

As a demonstration of ion-induced atomic mixing, we first show the change in composition of a very thin $\mathrm{Si}$ layer on top of a thick Pt film, during ion implantation and sputtering. As shown in Fig. 1, the virgin sample consists of a Si layer (380 $\AA$ ) sandwiched between an underlying Pt film (1800 $\AA$ ) and a thin surface layer of $\operatorname{Pt}(100 \AA)$. This thin-film structure was prepared by sequential depositions of $\mathrm{Pt}$ and $\mathrm{Si}$ (by using electron-gun evaporation) onto a clean $\mathrm{Si}$ wafer. The sample was then implanted by using $250 \mathrm{keV} \mathrm{Xe}$. The lower part of Fig. 1 shows that the surface layers were nearly uniformly mixed over a depth of $\sim 1000 \AA$, after a $\mathrm{Xe}^{+}$dose of $3 \times 10^{15}$ $\mathrm{cm}^{-2}$ had been implanted. Note that there are $\sim 4 \times 10^{17}$ atoms $/ \mathrm{cm}^{2}$ in this mixed surface layer.

There are other interesting points suggested by the data in Fig. 1. First, the underlying Pt-Si interface (i.e., that between the $\mathrm{Si}$ substrate and the thick Pt film) remains undisturbed, despite the mixing of Pt and Si near the surface region. This is consistent with previous observations that atomic mixing occurs only over a depth comparable to the ion range. Since the $\mathrm{Xe}$ ions did not penetrate through the underlying $\mathrm{Pt}-\mathrm{Si}$ interface, no intermixing occurred there. Second, the fact that the underlying interface remains sharp also indicates that no overall sample heating (due to the $\mathrm{Xe}^{+}$bombardment) occurred during the atomic mixing process, because $\mathrm{Pt}_{2} \mathrm{Si}$ formation at $\mathrm{Pt}-\mathrm{Si}$ interfaces occurs at $\sim 250^{\circ} \mathrm{C}$. Third, the atomic mixing process in this system is very efficient compared to sputter removal. In the lower portion of the figure, the shift of the rear edge of the $\mathrm{Pt}$ signal indicates that a dose of $10^{16} \mathrm{Xe}$ ions $/ \mathrm{cm}^{2}$ was required to sputter-remove $200 \AA$. Yet, only a third that dose was required to mix Pt and Si over depths five times greater. 


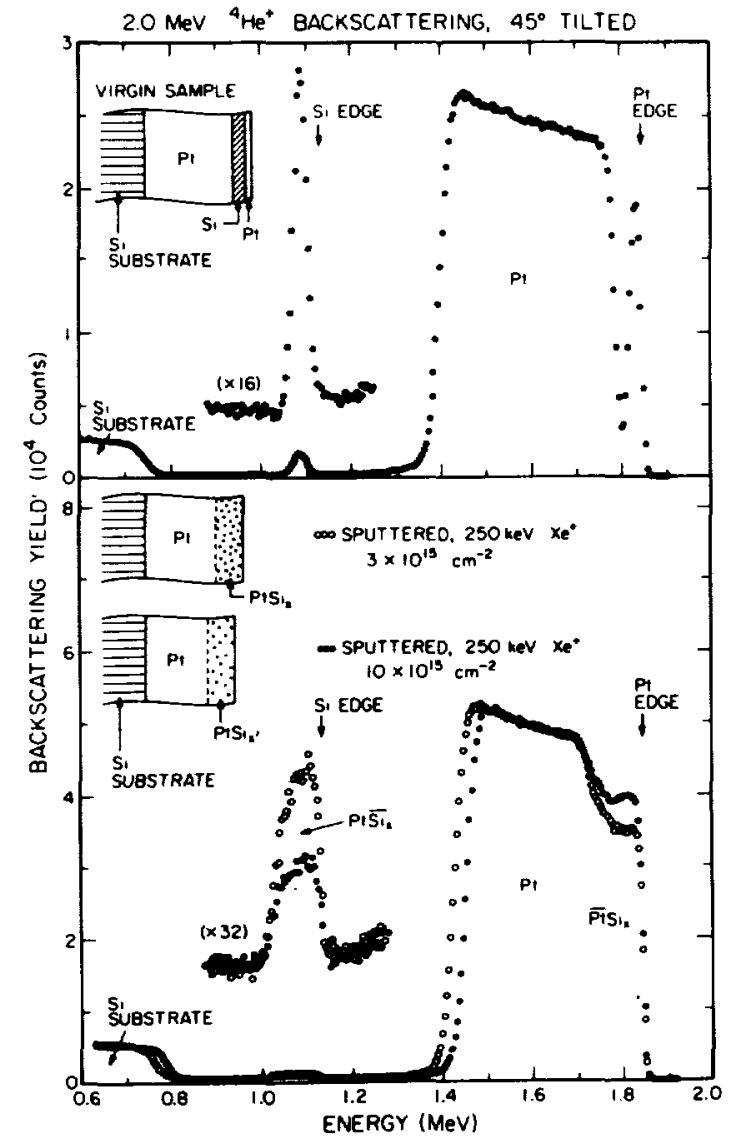

FIC. 1. Backscattering spectra of unsputtered and sputtered Pt-Si samples which show effects of ion-induced atomic mixing during sputtering.

This "highly efficient" mixing phenomenon has implications to many other related experiments. For example, thinfilm materials of any desirable compositions can possibly be produced by first depositing discrete layers of elemental materials followed by bombardment with a heavy ion beam of a suitable energy. Our recent experiments have shown that this technique can indeed produce thin-film materials with composition and structures which have not been obtained by conventional metallurgical means. ${ }^{12}$ This will be reported elsewhere. In the present paper, we shall concentrate on the effect of atomic mixing on sputtering and thin-film analysis.

\section{CHANGE OF THE SURFACE-LAYER COMPOSITION DURING SPUTTER ETCHING}

Since an $\mathrm{Xe}^{+}$dose $\$ 3 \times 10^{15} \mathrm{~cm}^{-2}$ was sufficient to mix the surface Pt-Si layers over a thickness comparable to the ion range, the mixing phenomenon can significantly affect the sputter-etching process as illustrated in Fig. 2. Because a much higher $\mathrm{Xe}^{+}$dose $\left(\sim 3 \times 10^{16} \mathrm{~cm}^{-2}\right.$ for a sputtering yield of 15$)$ will be required to sputter etch the same thickness, the mixing process can thus always have "ample time" to redistribute the remaining $\mathrm{Si}$-atoms uniformly over the thickness $W$. Thus, the Si-containing surface layer will appear to maintain a constant thickness, but with a decreasing Si-concentration because of the loss of Si atoms at surface due to sputter erosion.

This phenomenon has indeed been observed. One example is shown in the lower half of Fig. l. With a higher $\mathrm{Xe}^{+}$dose of $10 \times 10^{15} \mathrm{~cm}^{-2}$, more material was sputter etched. (Note the shift of the backedge of the Pt signal to higher energy position.) The signals of the Pt-Si mixed surface layer, however, maintained the same width, but with a drop in Si signal height (and a rise in the corresponding Pt signal height). In Fig. 1, the (near) surface composition as determined from the corresponding $\mathrm{Pt}$ and $\mathrm{Si}$ signal heights ${ }^{11}$ are $\mathrm{PtSi}_{0.66}$ and $\mathrm{PtSi}_{0.29}$, for the low and high $\mathrm{Xe}^{+}$dose samples, respectively. The numbers of $\mathrm{Pt}$ and $\mathrm{Si}$ atoms remained in the samples can be determined from the normalized integrated counts of $\mathrm{Pt}$ and Si signals. ${ }^{11}$ The amount of sputtering can thus be determined. This and the surface composition have been measured for each of the samples sputtered with $250 \mathrm{keV} \mathrm{Xe}{ }^{+}$to doses 3-20 $\times 10^{15} \mathrm{~cm}^{-2}$. The results are shown as the data points in Fig. 3. In Fig. 3, the sputtered thickness is obtained by assuming a constant atomic concentration of $6 \times 10^{22} \mathrm{~cm}^{-2}$ $\mathrm{Pt}$ and $\mathrm{Si}$ atoms. The parameter $\mathrm{W}$ is defined as the effective thickness of the surface layer, i.e., $W \equiv$ (total number of $\mathrm{Si}$ atoms in the surface layer)/(concentration of $\mathrm{Si}$ atoms at surface). The $W$ 's thus determined for all $\mathrm{Xe}^{+}$doses were nearly constant and could be represented by $W=700 \pm 30$ $\AA$.

Results similar to those of Pt-Si samples were also observed in $\mathrm{Al}-\mathrm{Au}$ samples as shown in Fig. 4. The Au distribution are observed to extend to a depth comparable to that of the implanted $\mathrm{Kr}$ distribution, while the Au concentration decreases with sputter etching.

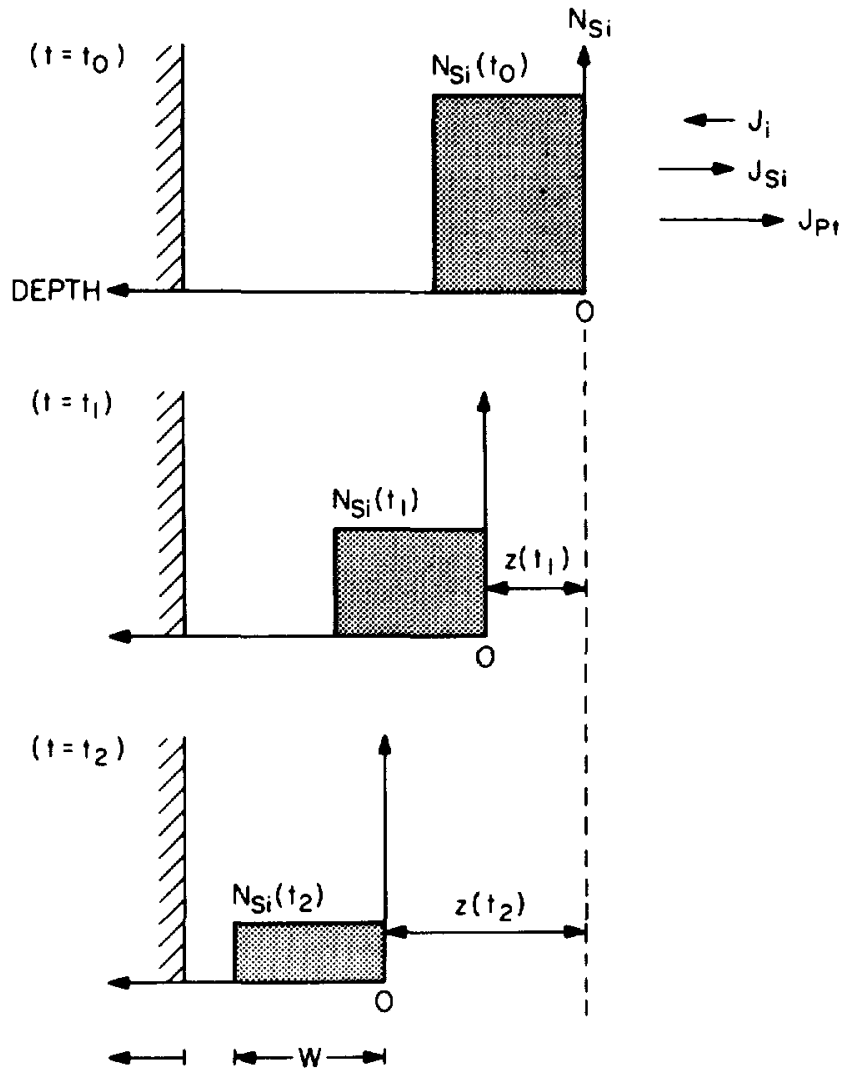

SUBSTRATE

Fic. 2. An illustration of the model for the change of surface Si concentration during sputtering. The main idea is the sputter-removal of $\mathrm{Si}$ and $\mathrm{Pt}$ atoms at surface and the redistribution of the remaining $\mathrm{Si}$ atoms over a constant depth. 


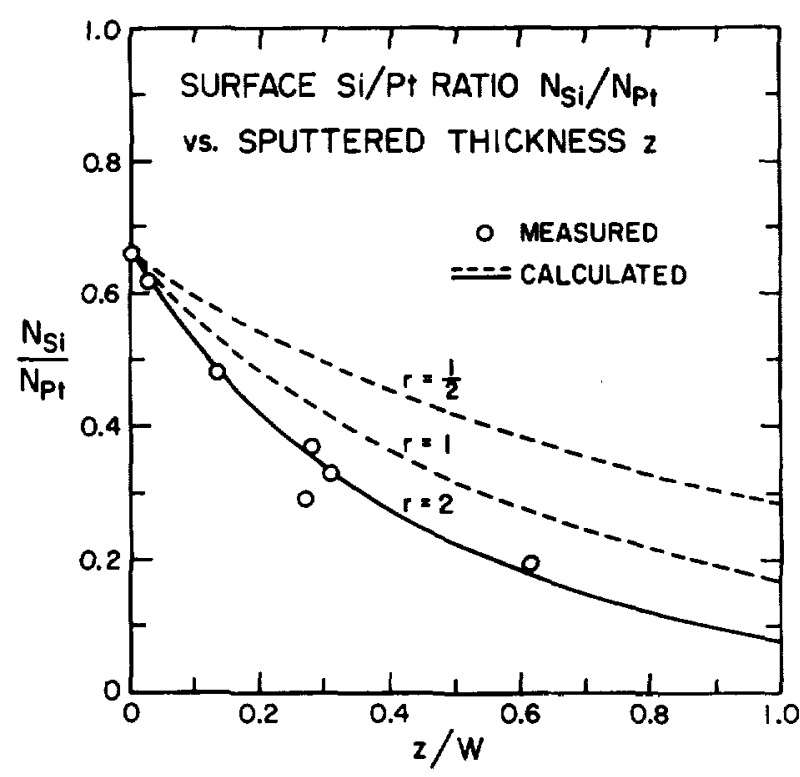

FIG. 3. Surface $\mathrm{Si} / \mathrm{Pt}$ concentration ratio as a function of the amount of material which has been sputter-removed. The curves are given by Eq. (6) which is derived from the model sketched in Fig. 2.

\section{PREFERENTIAL SPUTTERING}

Another phenomenon that affects thin-film analysis is the preferential sputtering of a compound material. ${ }^{10,11}$ The resultant surface composition changes have been observed by

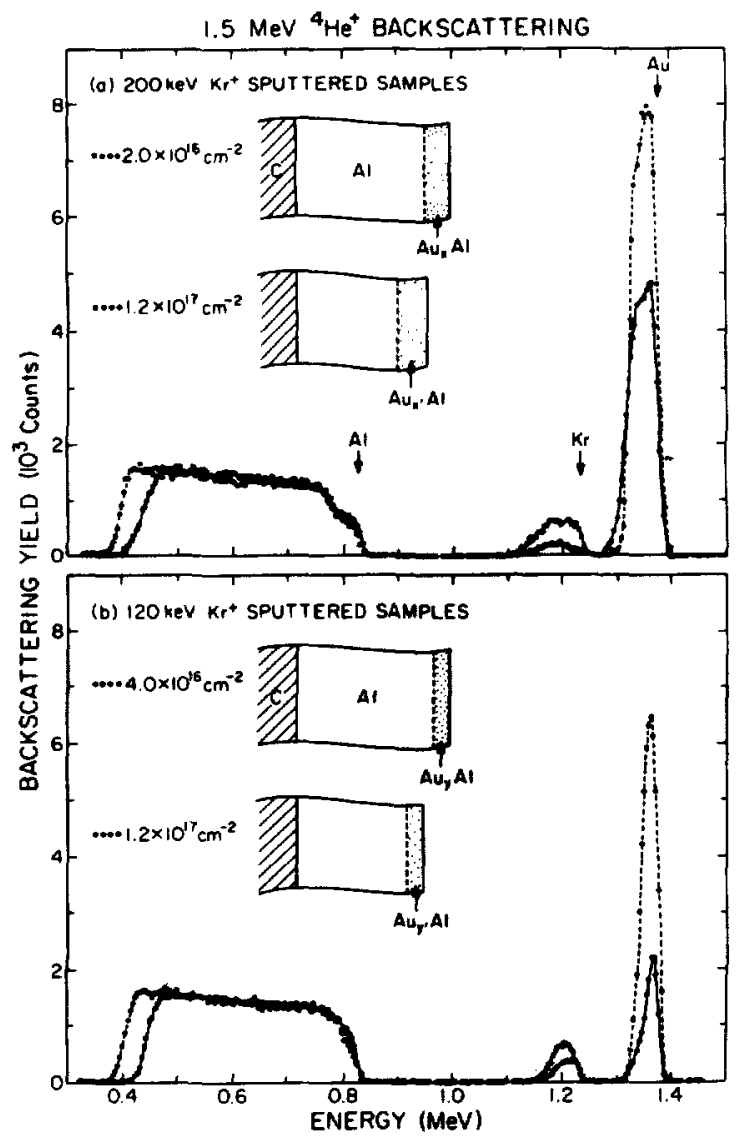

FIG. 4. Backscattering spectra of sputtered Al-Au samples which show the evolution of surface layers during sputtering. For each $\mathrm{Kr}^{+}$energy, the surface Au-Al-intermixed layer maintains a nearly constant thickness, which is comparable to the implanted $\mathrm{Kr}$ distribution.

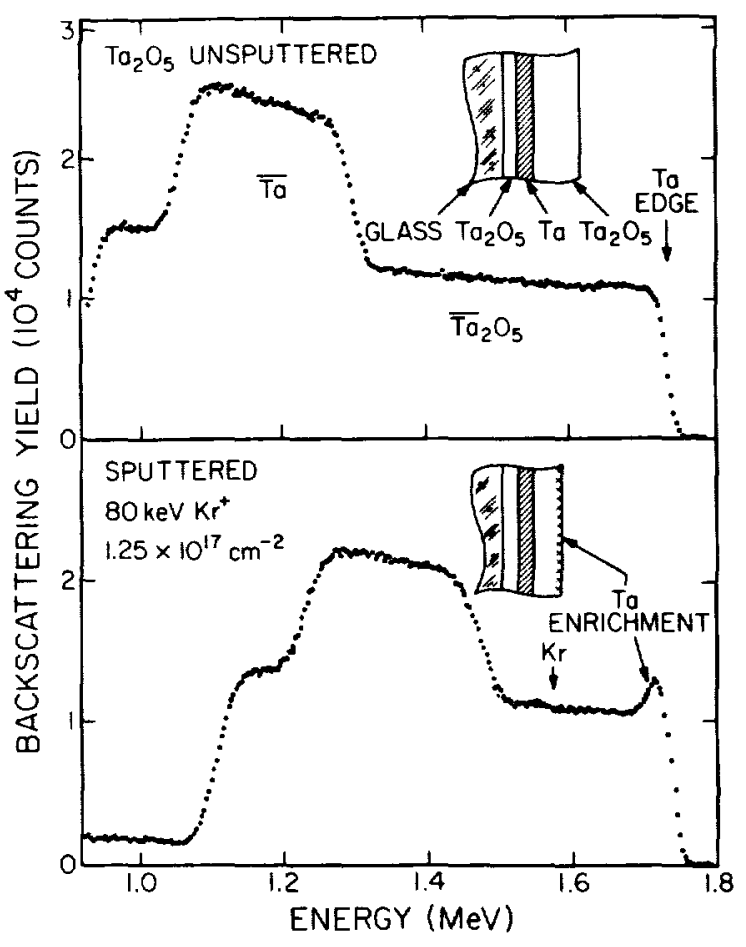

FiG. 5. Backscattering spectra of a $\mathrm{Ta}_{2} \mathrm{O}_{5}$ film before and after being sputtered by $80-\mathrm{keV} \mathrm{Kr}^{+}$. The unsputtered $\mathrm{Ta}_{2} \mathrm{O}_{5}$ was $\sim 6000 \mathrm{i}$ thick. After sputtering, a Ta-peak appears near the Ta signal edge, indicating a Ta enrichment in a surface layer of $\sim 600 \mathrm{i}$ thick.

numerous investigators. ${ }^{13}$ Figure 5 shows an example with the sputtering of a $\mathrm{Ta}_{2} \mathrm{O}_{5}$ film. ${ }^{10}$ In the sputtered sample, the near surface composition was determined to be $\sim \mathrm{Ta}_{4.6} \mathrm{O}_{5} .{ }^{10}$

Note the Ta-enriched surface layer in Fig. 5 has a thickness of $\sim 600 \AA$. This is again comparable to the ion range, but is much greater than the escape depth of the sputtered atoms $(\$ 10 \AA)$. Therefore, an ion-induced atomic mixing effect has been invoked in explaining the thicknesses of the altered surface layers. A phenomenological model based both on preferential sputtering at the surface and on atomic mixing over the ion range, has been developed to explain the formation kinetics of the altered surface layers ${ }^{11}$.

\section{MODEL FOR THE SURFACE LAYER COMPOSITION DURING SPUTTER ETCHING}

In Sec. III, the phenomenon was interpreted as due to the loss of Si-atoms at surface and the redistribution of the remaining Si atoms over a constant depth $W$, as illustrated in Fig. 2. Based on this concept, a model is developed in this section to relate the Si concentration to the amount of sputtering (i.e., to explain the data points in Fig. 3). This model takes preferential sputtering of $\mathrm{Si}$ into consideration and is quite similar to the one developed for the sputtering of PtSi. ${ }^{11}$

The conservation of $\mathrm{Si}$ atoms requires

$$
W \cdot d N_{\mathrm{Si}} / d t=-J_{\mathrm{Si}}
$$

where $N_{\mathrm{Si}}$ is the density of $\mathrm{Si}$ atoms (per unit volume) in the surface layer, $t$ is the sputtering time and $J_{\mathrm{Si}}$ is the magnitude 
of the flux of the sputtered $\mathrm{Si}$ atoms. $J_{\mathrm{Si}}$ is related to the incident ion flux $J_{i}$ and the total sputtering yield $S$, i.e.,

$$
J_{\mathrm{Si}}+J_{\mathrm{Pt}}=S J_{i},
$$

where $J_{\mathrm{Pt}}$ is the flux of sputtered $\mathrm{Pt}$ atoms. $J_{\mathrm{Si}}$ is also related to the surface $\mathrm{Si}$ concentration $N_{\mathrm{Si}}$ and

$$
J_{\mathrm{Si}} / J_{\mathrm{Pt}}=r \cdot\left(N_{\mathrm{Si}} / N_{\mathrm{Pt}}\right),
$$

where $N_{\mathrm{Pt}}$ is the surface $\mathrm{Pt}$ concentration and $r$ is the preferential sputtering factor. ${ }^{11}$

Equations (1), (2), and (3) can then be combined to obtain the values of $N_{\mathrm{Si}}$ during the sputtering process. For convenience, we define $x \equiv N_{\mathrm{Si}} / N_{\mathrm{Pt}}$ and $N_{0} \equiv N_{\mathrm{Si}}+N_{\mathrm{Pt}}$, which then give $N_{\mathrm{Si}}=N_{0} x /(1+x)$. Equations (2) and (3) then yield $J_{\mathrm{Si}}=S J_{i} \cdot r x /(r x+1)$. By using these new expressions for $N_{\mathrm{Si}}$ and $J_{\mathrm{Si}}$ and by assuming $N_{0}$ as a constant, Eq. (1) becomes

$$
N_{0} W \frac{d}{d t}\left(\frac{x}{1+x}\right)=-\frac{r x}{r x+1} S J_{i} .
$$

After some rearrangements Eq. (4) becomes

$$
\int_{x(0)}^{x(t)} \frac{\left(r x^{\prime}+1\right)}{r x^{\prime}\left(1+x^{\prime}\right)^{2}} d x^{\prime}=\frac{1}{N_{0} W} \int_{0}^{t} S J_{i} d t .
$$

By assuming $r$ as a constant, the integration on the lefthandside of Eq. (5) can be carried out by taking the partial fractions on the integrand. The integral on the right-hand side of Eq. (5) represents the total amount of material sputtered per unit area, up to time $t$. Therefore, $1 / N_{0} \int_{0}^{t} S J_{i} d t$ is the sputtered thickness which will be denoted as $z(t)$, as in Figs. 2 and 3. Equation (5) then becomes

$$
\left[\frac{r-1}{x+1}+\ln \left(\frac{x}{x+1}\right)\right]_{x(0)}^{x}=-\frac{z}{W / r}
$$

Equation (6) gives $z(x)$, which yields $z(x)$ upon inversion. The calculated $x(z)$ for $r=1 / 2,1$, and 2 are plotted in Fig. 3, which shows that $r=2$ yields better agreement with experiment. This is consistent with previous results ${ }^{11}$ on the sputtering of PtSi which showed a preferential sputtering of $\mathrm{Si}$ with $r \cong 2$. For $x(0) \ll 1$, Eq. (6) can be simplified to be

$$
x=x(0) e^{-z /(W / r)} \text {. }
$$

\section{INFLUENCE OF ATOMIC MIXING AND PREFERENTIAL SPUTTERING ON THIN-FILM AND INTERFACE ANALYSES-THE GENERALIZED MODEL}

In the previous sections the ion-induced atomic mixing phenomena have been investigated mostly on samples which contain a very thin layer of impurity atoms at surface. The situation is generally different in the depth profiling analyses. Usually, the impurity profiles (or thin-film structures) extend over depths much greater than the range (or $W$ ) of the ion used for sputtering, as illustrated in Fig. 6(a). To see the effects of atomic mixing and preferential sputtering on depth profiling, the model developed in Sec. $\mathrm{V}$ can be generalized to relate the apparent and the true impurity depth profiles (or thin-film structures).

Similar assumptions as those in Sec. $\mathrm{V}$ are made in this
(A)

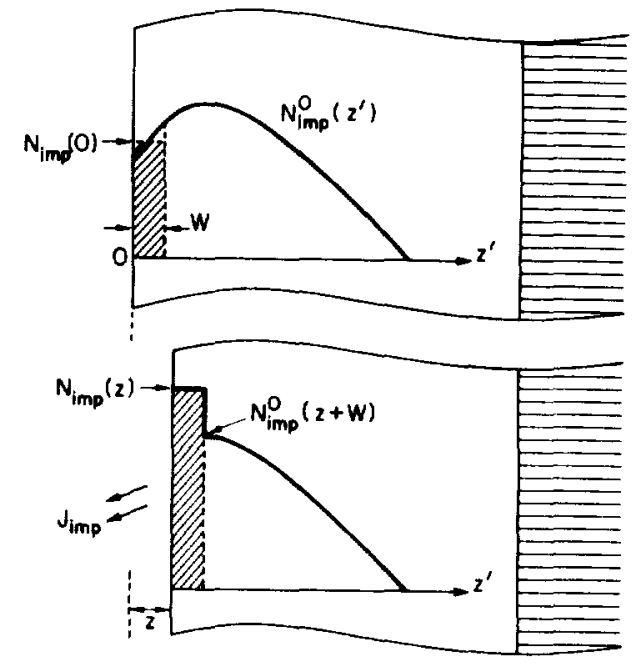

$W \cdot d N_{i m p}(z) / d t=-J_{i m p}+N_{i m p}^{0}(z+W) \cdot d z / d t$

(C)

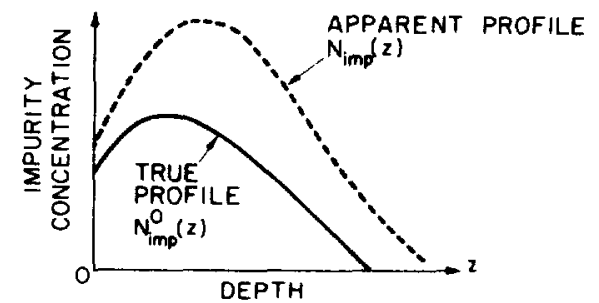

FIG. 6. An illustration of the influence of atomic mixing and preferential sputtering on the sputter-depth-profiling analysis.

calculation, i.e., that the impurity atoms influenced by ioninduced atomic mixing are always redistributed very "quickly" and uniformly over an effective thickness $W$, and that the rate of sputter-removal of impurity atoms is proportional to the surface impurity concentration multiplied by a constant preferential sputtering parameter $r$. The conservation of impurity atoms then requires

$$
W d N_{\mathrm{imp}}(z) / d t=-J_{\mathrm{imp}}+N_{\mathrm{imp}}^{0}(z+W) \frac{d z}{d t},
$$

where $z$ is the sputtered thickness, $N_{\text {imp }}(z)$ is the concentration of impurity atoms in the surface layer, $J_{\text {imp }}$ is the magnitude of the flux of sputtered impurity atoms and $N_{\mathrm{imp}}^{0}(z+$ $W$ ) is the concentration of the original (true) impurity distribution at the depth of $z+W$ [(see Fig. 6(b)]. Since it is the surface concentration $N_{\text {imp }}(z)$ that is measured as a function of sputtered thickness $z$ in the depth profiling techniques, $N_{\text {imp }}(z)$ will become the measured (or "apparent") impurity depth profile |See Fig. 6(c)]. Equation (8) is similar to Eq. (1) except for the term $N_{\mathrm{imp}}^{0}(z+W) \cdot d z / d t$, which represents the supply of impurity atoms from the back-side of the surface layer due to the ion-induced atomic mixing effect. For simplicity, we consider first the cases of very low impurity concentrations, i.e., $x_{0}(z) \equiv N_{\text {imp }}^{0}(z) / N_{0} \ll 1$. Equation (8) then becomes

$$
W d x(z) / d z=-r x(z)+x_{0}(z+W),
$$

where $x(z) \equiv N_{\text {imp }}(z) / N_{0}$. The relation $J_{\text {imp }} \cong r x(z) N_{0} d z / d t$

J. Vac. Scl. Technol., Vol. 16, No. 2, Mar./Apr. 1979 
has been used in obtained Eq. (9). With constant $r$ and W, Eq. (9) can be solved ${ }^{14}$ to yield:

$$
\begin{aligned}
x(z)=\int_{0}^{z} e^{-r\left(z-z^{\prime}\right) / W} & x_{0}\left(z^{\prime}+W\right) d z^{\prime} / W \\
& +e^{-r z / W} \frac{1}{W} \int_{0}^{W} x_{0}\left(z^{\prime}\right) d z^{\prime}
\end{aligned}
$$

Equation (10) relates the measured profile $x(z)$ to the true profile $x_{0}(z)$.

Equation (10) can be interpreted in physical terms. The original true profile $x_{0}(z)$ can be considered as being composed of many infinitesimal sections each of thickness $d z^{\prime}$. Equation (7) predicts that each infinitesimal section would generate an apparent profile of $\left[x_{0}\left(z^{\prime}\right) d z / W\right] e^{-r\left(z-z^{\prime}\right) / W}$ for $z>z^{\prime}$. Therefore, the total apparent profile $x(z)$ would be the superposition of contributions from all infinitesimal sections, i.e., $x(z)=\int_{0}^{z} e^{-r\left(z-z^{\prime}\right) / W} x_{0}\left(z^{\prime}\right) d z^{\prime} / W$. However, this is still not the same as Eq. (10). The discrepancy is due to the assumption of the "very fast" atomic mixing over the thickness $W$. Consider, for example, the infinitesimal section at $z^{\prime}=$ $W^{+}$. As soon as this section gets in touch with the backside of the surface layer, the atomic mixing effect will very quickly spread out all the impurity atoms in that infinitesimal section over the thickness $W$. Therefore, the infinitesimal section at $z^{\prime}=W^{+}$will contribute to $x(z)$ as if it were at $z^{\prime}=O^{+}$. This accounts for $x_{0}\left(z^{\prime}+W\right)$, instead of $x_{0}\left(z^{\prime}\right)$, in the first integral of Eq. (10). It also explains the necessity of the second integral in Eq. (10).

Equation (10) can be used to calculate the apparent profile $x(z)$ for any given true profile $x_{0}(z)$. A few examples are shown in Figs. 7 and 8 . With a given magnitude of $W$, Fig. 7 also illustrates the effects of various preferential sputtering factor $r$, in profiling a uniform layer on top of a substrate. Without preferential sputtering (i.e., $r=1$ ), the apparent profile is identical to the true one until the ion beam begins to reach the interface. Then, the atomic mixing smears out the interface over a distance characterized by $W$. After that, the mixing phenomenon cause an exponential tail of the impurity into the substrate. With preferential sputtering, the impurity atoms become either enriched or depleted in the surface layer. In the beginning, it takes an amount of sput-

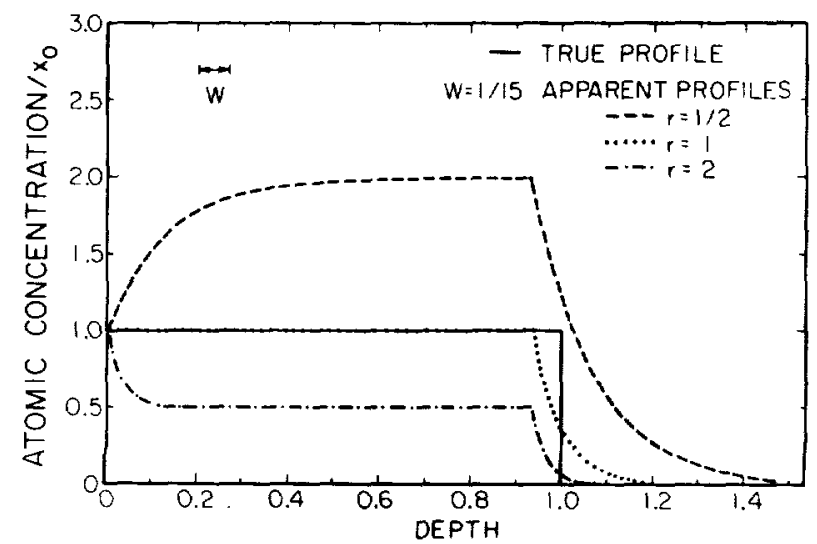

FIG. 7. Calculated apparent profiles of a true profile of a square shape. With a constant $W$, the apparent profiles were calculated for various preferential sputtering conditions by using Eq. (10).

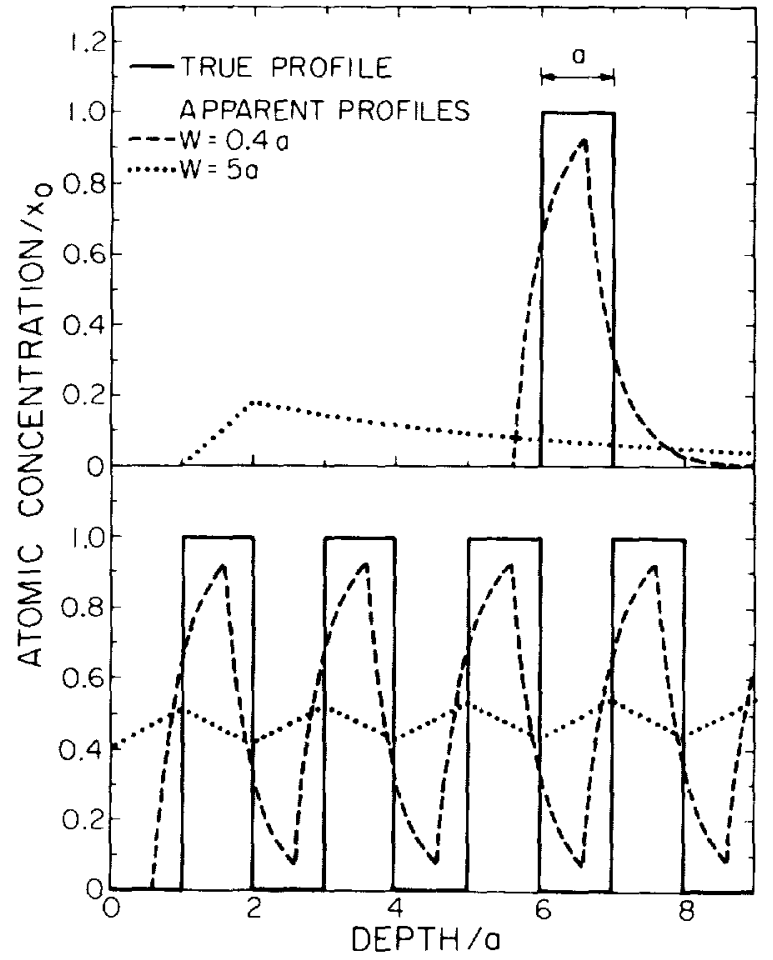

Fic. 8. Further examples of calculated apparent profiles illustrating the effect of $\boldsymbol{W}$ in depth-profiling measurements. These calculations assumed no preferential sputtering (i.e., $r=1$ ).

tering (a sputtered thickness characterized by $W / r$ ) for the surface composition to reach the steady state. The interface is smeared out over a distance of $\sim W / r$.

Figure 8 shows the effects of $W$ on a buried layer and a periodic structure. For a buried layer of thickness $a$, the depth-profiling more or less reproduce the true profile if $W$ $\ll a$. For $W>a$, the profile is much distorted. The ion beam can "foresee" the impurity long before the real depth is actually reached. This translates into a loss of "resolution" in the profiling of a periodic structure as shown in the lower half of Fig. 8.

For practical purposes, it is of interest to deduce $x_{0}(x)$ from known $x(z)$, because $x_{0}(x)$ is the purpose of the measurement and $x(z)$ is what actually measured. With known $x(z)$, Eq. (9) can be used to obtain $x_{0}(x)$ for $z>W$. However, it appears impossible to know $x_{0}(z)$ for $0<z<W$. Again, this is due to the "fast" atomic mixing process, which completely mixed up the surface layer before the measurements. Only the average value of the surface layer can be obtained, i.e., $x(0)=$ $1 / W \int_{0}^{W} x_{0}\left(z^{\prime}\right) d z^{\prime}$. [See Fig. 6(a)].

\section{DISCUSSION}

For the purpose of studying the mixing phenomena with RBS techniques, ion energies $10-300 \mathrm{keV}$ have generally been used to produce a mixed layer of a few hundred $\dot{A}$. There are questions about whether the phenomena will be similar for lower energies $(0.5-10 \mathrm{keV})$, which are actually used for AES and SIMS analyses. One way to answer this question is to study an energy dependence of the mixing phenomena. Indeed, RBS experiments using glancing angles were able to show a mixed layer of $\sim 100 \AA$ in $\mathrm{PtSi}$ as a result of $10-\mathrm{keV} \mathrm{Ar}^{+}$ 
sputtering. " That the mixed-layer thickness $(W)$ is nearly proportional to the ion energy has been demonstrated for $\mathrm{Ar}^{+}$ energies of 10-160 keV. ${ }^{11}$ By extrapolating from that data, a mixed-layer thickness of $\sim 30 \AA$ is predicted for $2-\mathrm{keV} \mathrm{Ar}{ }^{+}$. In fact, AES and SIMS experiments of Chuang and Wandelt, ${ }^{9}$ Wittmaack et al., ${ }^{8}$ and Ishitani et al. ${ }^{7}$ have shown interface broadening similar to that addressed in the present paper. The thicknesses of the mixed layers and their energy-dependence obtained by these authors are consistent with RBS results.

There have been other experiments which demonstrate the ion-induced atomic mixing effects. Using SIMS, Schulz et al ${ }^{3}$ and $\mathrm{McHugh}^{4}$ have observed that the tails or broadening of impurity profiles are related to the energy of the sputtering ion. With RBS, van der Weg et al. ${ }^{15}$ and Poate et al. ${ }^{16}$ have observed interfacial mixing (or reaction) when the implanted Ar-range can reach the interface between a surface metal layer and the underlying Si substrate. With RBS, Hart et al. ${ }^{5}$ and Wach and Wittmaack ${ }^{6}$ have demonstrated mixing of surface contaminants into depths comparable to the ion range. Furthermore, our previous RBS study of the preferential sputtering has shown that the surface layer composition changes are generally observed (in various materials) with the thickness of the altered surface layer always comparable to the ion range. ${ }^{10,11} \mathrm{We}$ believe that all these experiments demonstrate similar effects of the ion-induced atomic mixing.

While the phenomenon seems to be quite universal, there are some systems which appear to be less influenced by ioninduced atomic mixing. For example, much less ion-induced interfacial reactions were observed with $\mathrm{Mo}^{17}$ and $\mathrm{Nb}^{18}$ films on $\mathrm{Si}$ than with $\mathrm{Pd}^{15}$ and $\mathrm{Pt}^{16}$ films on $\mathrm{Si}$ substrate. It seems that those materials which require higher doses for atomic mixing also have lower sputtering yields. The fact that ion mixing and sputtering have some common features (i.e., both of them are related to the density of collision cascade and the atomic binding energy in the material) has recently been experimentally demonstrated. ${ }^{12}$ On the other hand, if lowsputtering yield always accompanies low ion-mixing rate, the ion-mixing process will always have "ample time" to create a mixed layer like that described in Section III. The thickness of the mixed layer will depend on the relative efficiency of mixing and sputtering. Roughly speaking, the mixed layer has a characteristic length of $D / v$, where $D$ is the effective diffusivity in the ion-mixing process and $v$ is the surface receding velocity due to sputtering. If $D / v$ is much larger than the mixing range $W$ (which is comparable to the ion range), then the thickness of the mixed layer is limited by $W$ rather than by $D / v$.

In addition to the $D / v$ argument, the observation that $W$ is nearly constant in the Pt-Si system (in the present paper) may not be as good as approximation in other systems. Since the composition of the surface layer is changing during sputtering, the ion range and the effectiveness of the ioninduced atomic mixing can have corresponding variations. The fact that $\mathrm{Si}$ was used as the diluted alloying element in $\mathrm{Pt}$ could have simplified the problem because of the fact that $\mathrm{Si}$ is much lighter than $\mathrm{Pt}$. The $\mathrm{Pt}$ atoms would then be responsible for triggering most of the collisions in the cascade. Therefore, the changing Si-concentration would not signifi- cantly affect the ion range and atomic mixing. This may have helped in maintaining the constancy of $W$.

\section{SUMMARY.}

The influence of atomic mixing on profiling of metalsemiconductor interfaces is summarized in Fig. 9 which depicts the penetration of one Ar ion into a sample during the sputtering process. The size of the arrows marked Si or Pt illustrate the relative ion yield. When the ion approaches within $W$ of the interface, atomic mixing occurs. Since $W$ is greater than the escape depth of Auger electrons, AES measurements will indicate the presence of a broad interface containing a mixture of $\mathrm{Pt}$ and $\mathrm{Si}$ rather than a sharp transition. With further sputtering, the $\mathrm{Pt}$ will be mixed deeper in the $\mathrm{Si}$ and AES measurements will show a tail in the distribution of $\mathrm{Pt}$.

One can reduce the apparent width of the interface by reducing the energy of the sputtering ions, by use of heavy ions or by use of glancing angles. However, the escape depth of Auger electrons is usually less than $10 \AA$, a value less than $W$ in all but extreme cases. Consequently, we believe that sputter sectioning is not a suitable technique to determine the composition of sharp interfaces in metal-semiconductor systems.

\section{ACKNOWLEDGMENTS}

The authors are indebted to their colleagues: G. E. Chapman, S. E. Matteson, J. K. Hirvonen (Naval Research Lab.),
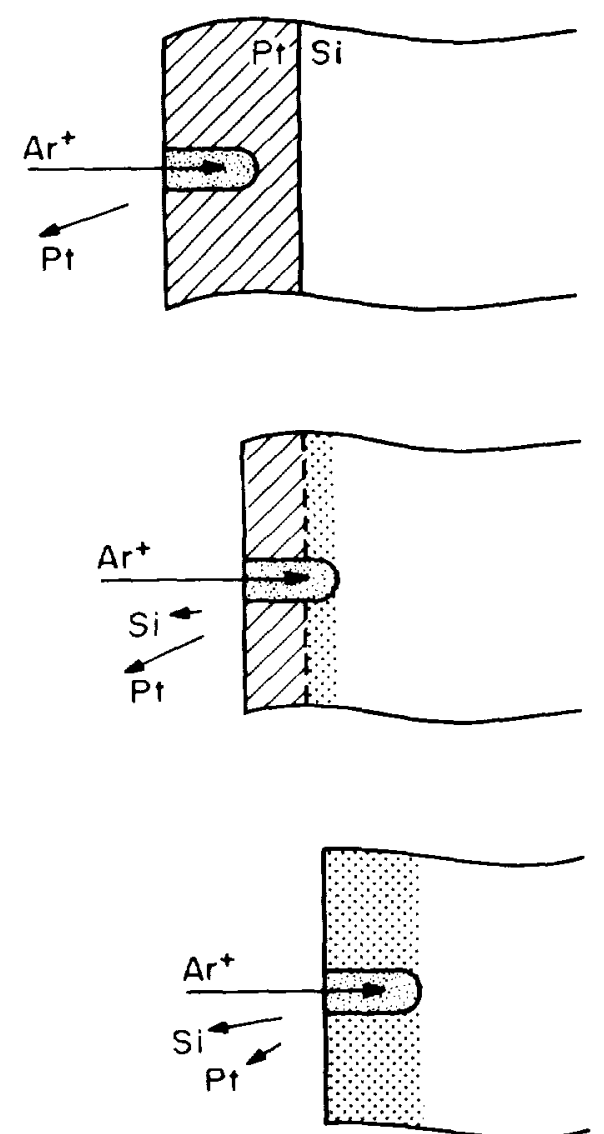

FiG. 9. A schematic picture showing effects of atomic mixing on the sputter depth profiling through an interface.

J. Vac. Scl. Technol., Vol. 16, No. 2, Mar./Apr. 1979 
and I. M. Poate (Bell Labs), for their interest and participation in part of the present experimental work. They are also indebted to D. M. Scott, whose work on the accelerator made most data in this paper possible, and to J. J. Mallory for his skillful sample preparations. The partial financial support of the Army Research Office is gratefully acknowledged.

IJ. S. Williams, Nucl. Instrum. Methods 149, 207 (1978).

${ }^{2}$ See, for example, A. Joshi, L. E. Davis, and P. W. Palmberg, in Methods of Surface Analysis, edited by A. W. Czanderna (Elsevier, Amsterdam, 1975), Chap. 5

${ }^{3}$ F. Schulz, K. Wittmaack, and J. Maul, Rad. Effects 18, 211 (1973).

4. A. McHugh, Rad. Effects 21, 209 (1974)

${ }^{5}$ R. R. Hart, H. L. Dunlap, and O. J. Marsh, J. Appl. Phys. 46, 277 (1975)

${ }^{6} \mathrm{~W}$. Wach and $\mathrm{K}$. Wittmaack, Nucl. Instrum. Methods 149, $259(1978)$

${ }^{7}$ T. Ishitani, R. Shimizu, and H. Tamura, Appl. Phys. 6, 277 (1975).
${ }^{8} \mathrm{~K}$. Wittmaack, paper presented at the 8th International Conference on X-Ray Optics and Microanalysis, Boston, MA, August, 1977.

${ }^{9}$ T. J. Chuang and K. Wandelt, IBM J. Res. Develop. 22, 277 (1978).

${ }^{10}$ Z. L. Liau, W. L. Brown, R. Homer, and J. M. Poate, Appl. Phys. Lett. 30, 626 (1977).

1'Z. L. Liau, J. W. Mayer, W. L. Brown, and J. M. Poate, J. Appl. Phys. 49, 5295 (1978).

${ }^{12}$ B. Y. Tsaur, Z. L. Liau, and J. W. Mayer (unpublished).

${ }^{13}$ See, for example, the references listed in Ref. 11.

${ }^{14}$ See, for example, F. B. Hildebrand, in Advanced Calculus for Applications (Prentice-Hall, New York, 1962), Chap. 1.

${ }^{15}$ W. F. van der Weg, D. Sigurd, and J. W. Mayer, in Applications of Ion Beams to Metals, edited by S. T. Picraux, E. P. EerNisse, and F Vook (Plenum, New York, 1974), p. 209.

16J. M. Poate and T. C. Tisone, Appl. Phys. Lett. 24, 391 (1974)

${ }^{17}$ H. Nishi, T. Sakurai, T. Akamatsu, and T. Furuya, Appl. Phys. Lett. 25, 337 (1974).

18. E. Matteson, J. Roth and M-A. Nicolet, paper presented at the International Conference on lon Beam Modification of Materials, Budapest, Hungary, September, 1978. 